\title{
A influência midiática para automedicação do novo coronavírus: revisão literária
}

\author{
The media influence on self-medication of the new coronavirus: a literature review \\ La influencia mediática para la automedicación del nuevo coronavirus: una reseña literaria
}

Recebido: 24/09/2021 | Revisado: 30/09/2021 | Aceito: 02/10/2021 | Publicado: 04/10/2021

Juliana Matos Gomes Ruiz

ORCID: https://orcid.org/0000-0002-0821-4615

Faculdade de Palmas, Brasil

E-mail: julianamg9@hotmail.com

Érica Ferreira de Souza

ORCID: https://orcid.org/0000-0003-4274-027X

Faculdade de Palmas, Brasil

E-mail: ericasouzarodriguee@gmail.com

Maykon Jhuly Martins de Paiva

ORCID: https://orcid.org/0000-0002-6228-4550

Faculdade de Palmas, Brasil

E-mail: maykonjhuly@hotmail.com

\begin{abstract}
Resumo
A automedicação e uma hábito adotado pelos idivíduos que em muitas vezes acabam optando por este método em busca de uma alívio rápido para seus males. No final de 2019 o SARS-CoV-2 foi descoberto após casos registrados na China, com relatos que incluíam sintomas respiratórios leves e persistentes característicos da COVID-19. É notório que diante deste cenário houve uma grande influência da mídia para o uso de medicamentos como um suposto tratamento imediato, mesmo não havendo uma eficácia comprovada cientificamente. $\mathrm{O}$ aumento da automedicação é algo histórico em toda a humanidade, pessoas doentes que têm pouco acesso a estudos científicos e saúde de qualidade constantemente optam por se automedicarem sem prescrição médica. Durante a pandemia do novo coronavírus, várias falsas notícias apontam medicamentos milagrosos para tratar a doença da COVID - 19, porém, são cientificamente ineficazes e pouco provados. $\mathrm{O}$ uso de medicamentos sem prescrição médica é um risco para a saúde de quem costuma aderir essas práticas. Este artigo de revisão literária tem como objetivo geral identificar os motivos da automedicação durante a pandemia do coronavírus e os principais riscos da utilização de medicamentos sem prescrição médica.
\end{abstract}

Palavras-chave: Coronavírus; Mídia; Automedicação.

\begin{abstract}
Self-medication is a habit adopted by individuals who often end up opting for this method in search of a quick relief for their ailments. In late 2019 SARS-CoV-2 was discovered after cases were reported in China, with reports that included mild and persistent respiratory symptoms characteristic of COVID-19. It is notable that against this backdrop, there has been a great deal of media influence for the use of medication as a supposed immediate treatment, despite no scientifically proven efficacy. The increase in self-medication is something historical throughout humanity, sick people who have little access to scientific studies and quality healthcare constantly opt for self-medication without a prescription. During the pandemic of the new coronavirus, several false news reports point to miracle drugs to treat the COVID-19 disease, but they are scientifically ineffective and poorly proven. The use of over-the-counter medications is a health risk for those who frequently adhere to these practices. This literature review article has the overall objective of identifying the reasons for self-medication during the coronavirus pandemic and the main risks of over-the-counter medication use.
\end{abstract}

Keywords: Coronaviruses; Media; Self-medication.

\section{Resumen}

La automedicación es un hábito que adoptan las personas que muchas veces acaban optando por este método en busca de un alivio rápido de sus dolencias. A finales de 2019, se descubrió el SARS-CoV-2 luego de casos reportados en China, con reportes de que incluyó síntomas respiratorios leves y persistentes característicos de COVID-19. Es notorio que, ante este escenario, hubo una gran influencia de los medios de comunicación para el uso de la medicación como un supuesto tratamiento inmediato, aunque no existe una eficacia científicamente probada. El aumento de la automedicación es algo histórico en toda la humanidad, los enfermos que tienen poco acceso a los estudios científicos y una salud de calidad optan constantemente por automedicarse sin receta. Durante la nueva pandemia de coronavirus, varios informes falsos apuntan a medicamentos milagrosos para tratar la enfermedad del COVID- 19, sin embargo, son científicamente ineficaces y poco probados. El uso de medicamentos de venta libre es un riesgo para la salud de quienes 
habitualmente se adhieren a estas prácticas. Este artículo de revisión de la literatura tiene como objetivo identificar los motivos de la automedicación durante la pandemia de coronavirus y los principales riesgos del uso de medicamentos de venta libre.

Palabras clave: Coronavirus; Medios de comunicación; Automedicación.

\section{Introdução}

A pandemia causada pelo coronavírus vem sendo um grande problema para os sistemas de saúde de todos os países. O novo vírus descoberto em 2019 vem chamando atenção de todas as grandes nações, causando medo e receio em toda população mundial (Freitas, Napimouga \& Donalisio, 2020). De acordo com a Organização Mundial da Saúde (OMS), no ano de 2020 foram registrados em média 3 milhões de mortes pela COVID - 19.

Nesse sentido, a sociedade em geral vem procurando formas e medicamentos para se tratar do vírus. Segundo Silva e Gonzales (2021), durante a pandemia a população vem se automedicando com o intuito de diminuir os sintomas da doença causada pelo vírus da COVID - 19, entretanto, a automedicação pode provocar efeitos indesejados ao usuário. Nesse sentido, Guimarães e Carvalho (2020) abordam que um dos principais potencializadores para a automedicação são as abordagens de alguns líderes mundiais que buscam uma solução rápida e barata, porém cientificamente ineficaz, causando na sociedade até mesmo um comportamento negacionista contra a ciência.

Os principais medicamentos que vêm sendo utilizados durante a pandemia são: Hidroxicloroquina, Ivermectina e a Azitromicina. Medicamentos esses que são ineficazes de acordo com a OMS (Silva \& Gonzales., 2021). Imoto et al. (2020), constata em sua pesquisa que não existem dados suficientes que comprove a eficácia da cloroquina e da hidroxicloroquina contra o novo coronavírus, além de que os pacientes com COVID - 19 que utilizarem tais medicamentos de forma inadequada podem até mesmo prejudicar a própria saúde.

O papel do farmacêutico durante a pandemia se faz extremamente importante pois de acordo com Cardoso et al. (2021), os farmacêuticos têm um papel fundamental durante a pandemia, visto que sua orientação direcionada pode evitar que pacientes com o vírus façam o uso incorreto de medicamentos. A Assistência Farmacêutica durante a pandemia da COVID - 19 é de extrema importância, no controle da transmissão da doença e na atenção às necessidades da população, de forma a promover o uso racional de medicamentos durante a crise (Rubert, Deuschle \& Deuschle 2021).

\section{Metodologia}

Esse estudo se caracteriza como uma pesquisa bibliográfica que, segundo Gil (2002) é o estudo desenvolvido com base em materiais já elaborados, constituídos principalmente por livros e artigos científicos. Pizzani, Silva, Bello e Hayashi (2012), consideram a pesquisa bibliográfica importantíssima para a construção do conhecimento do pesquisador, portanto, é importante que se tenha dedicação e atenção por parte de quem deseja utilizar desse método.

A pesquisa foi realizada através da análise de materiais e documentos, tendo como obetivo principal a abordagem sobre o impacto da automededicação durante a pandemia da COVID-19 por meio de fake news.

Foram utilizados como critério de inclusão os seguintes aspectos: Artigos científicos e livros pertinente à temática; estudos contendo os seguintes descritores: pandemia, automedicação e saúde.

Como critério de exclusão, artigos que apresentaram: repetidos e resenha de livros e que não correspondiam ao estudo em questão. Desta forma, foram utilizados 20 artigos nas bases de dados indexados no Quadro 1: 
Quadro 1 - Identificação dos artigos a partir da revista, autores e ano de publicação.

\begin{tabular}{|l|l|l|}
\hline Revista & Autores & Ano \\
\hline Medicina S/A & Trotta & 2020 \\
\hline BRASIL & Comunicação do CFF & 2020 \\
\hline Revista Agência Senado & Guedes & 2017 \\
\hline Revista De Extensão Do Instituto Federal Catarinense & Wiese, et al. & 2020 \\
\hline Cadernos De Saúde Pública, & Melo, et al. & 2021 \\
\hline Interamerican Journal Of Medicine And Health & Carvalho, Guimarães & 2020 \\
\hline Brazilian Journal Of Development & De Fátima Souza, et al. & 2021 \\
\hline Revista Brasileira De Ciências Da Vida, & Oliveira et al. & 2017 \\
\hline Brazilian Journal Of Development & Ferreira; De Carvalho & 2021 \\
\hline Brazilian Journal Of Development & Dos Santos, et al. & 2021 \\
\hline Revista Eletrônica De Comunicação, Informação E Inovação Em Saúde & Falcão, De Souza, & 2021 \\
\hline Epidemiologia E Serviços De Saúde & Freitas, et al.. & 2020 \\
\hline Cadernos De Saúde Pública, & Melo, et al. & 2021 \\
\hline Revista De Extensão Do Instituto Federal Catarinense & Wiese, et al. & 2020 \\
\hline Comunicação Em Ciências Da Saúde & Imoto, et al. & 2020 \\
\hline Revista: Pub Saude & Miguel: De carvalho, & 2021 \\
\hline Scientific Electronic Library Online (Scielo) & Naves, et al. & 2010 \\
\hline RDBCI: Revista Digital De Biblioteconômia E Ciência Da Informação & Pizzani, et al. & 2012 \\
\hline
\end{tabular}

Fonte: Autores (2021).

\section{Resultados e Discussão}

A automedicação é um hábito social e não apenas farmacológico pelo fato de se manifestar a partir de comportamentos sociais de determinados grupos (Naves, Castro, Carvalho \& Merchán-Hamann, 2010). De acordo com Amaral, Veiga, Nelas, Coutinho e Chaves. (2019) a automedicação é um grande problema para a saúde e constitui um desafio para diversos países. Wiese et al. (2020), abordam que o livre acesso aos medicamentos sem receita ou prescrição médica contribui para essa cultura podendo causar grandes problemas de saúde a quem adere a essa prática.

Segundo Ferreira e Carvalho, (2021) fatores adversos contribuem para que as pessoas realizem a automedicação, exemplos como uma jornada de trabalho intensa, rotina familiar e estudantil acabam a limitar o tempo, tornando necessária uma busca de alívio rápido para certas enfermidades, desta forma a população acaba por se automedicar no intuito de não desperdiçar tempo e atrapalhar as suas atividades cotidianas. A mídia acaba exercendo uma grande influência sobre os indivíduos em relação à automedicação, quando se trata de medicamentos isentos de indicação médica, já que as indústrias farmacêuticas utilizam as propagandas como forma estratégica para o aumento lucrativo, que por muitas vezes visando apenas a venda dos seus fármacos acabam negligenciando informações importantes para o consumidor, como reações adversas e contra indicações dos fármacos. 
Medicamentos são compostos químicos que quando utilizados de forma adequada tem a finalidade de prevenir, reverter ou atenuar quadros patológicos. No entanto, quando utilizados de forma incorreta ou que não haja o conhecimento necessário o efeito pode ser contrário, diante disto se torna notório a importância de um profissional de saúde, que possua os conhecimentos adequados, na orientação ao indivíduo que nessecite do uso de medicamentos (Miguel \& Carvalho, 2021).

Com o surgimento da pandemia causada pelo novo coronavírus a alto medicação tornou-se algo mais frequente, devido à busca incessante da população não apenas por medicamentos relacionados ao tratamento da COVID-19 mas, também para tratar problemas secundários relacionados ao isolamento social. O profissional farmacêutico destaca-se dentro de todo o cenário da automedicação e seus agravantes, com atuação fundamental na identificação dos erros e agravos gerados por essa prática. Atuando na promoção da educação em saúde, prestando esclarecimentos a respeito da eficácia e segurança na administração de fármacos, assim como na prevenção dos riscos da automedicação à saúde (Bispo 2020).

Silva e Gonzales (2021), em seu estudo relatam a importância do farmacêutico para controlar e orientar a população sobre os métodos ideais para tratamento da COVID - 19, fazendo com que diminua o alto índice de automedicação neste período de pandemia. Gomes, Rocha, Viana e Bachur (2018) aborda que os meios mediáticos são grandes potencializadores para o consumo desenfreado de medicamentos. Desta forma, Carvalho e Guimarães (2020) relatam que a internet é um importante mecanismo de notícias durante a pandemia, entretanto, tornou- se uma grande contribuidora para o compartilhamento de notícias falsas podendo causar grandes problemas à população, principalmente aqueles que detém de pouco conhecimento científico.

Esse fenômeno de produção, compartilhamento de notícias falsas e desinformação, chamado de "infodemia", afeta, principalmente, os cidadãos desprovidos de senso crítico e de alfabetização digital e que, assim, costumam colocar em prática o que leem na internet. A velocidade com que as notícias e a grande quantidade de matérias que chegam aos indivíduos agravam mais ainda esse cenário (Carvalho \& Guimarães, 2020, p.2).

Durante a pandemia iniciada em 2019, o uso de automedicação vem sendo um assunto bastante discutido na internet por meio das redes sociais, os denominados "Kit COVID" ou "tratamento precoce" não são nada mais do que medicamentos sem nenhuma eficácia cientificamente comprovada (Melo, Duarte, Moraes, Fleck \& Arrais, 2021). Diante da busca incessante por medicamentos que possam tratar o novo coronavírus, a população tende a buscar soluções rápidas para o problema.

Um dos principais medicamentos divulgado pela mídia e até mesmo por representantes mundiais como a possível "cura" do vírus é a hidroxicloroquina (HCQ), que de acordo com Santos, Monteiro, Sousa e Araújo (2021) se trata de um medicamento sem eficácia cientificamente comprovada, podendo causar vários problemas de saúde pois se trata de um produto extremamente tóxico. Melo et al. (2021) abordam que a gigantesca utilização de medicamentos sem o uso de prescrições médicas pode ser associada aos diversos estímulos midiáticos e de autoridades. Diante de todo esse cenário de utilização desenfreada da HCQ, a Agência Nacional de Vigilância Sanitária por meio da resolução RDC no 351 que entrou em vigor em março de 2020 , faz com que esse medicamento seja vendido apenas por meio de uma Receita de Controle Especial.

Para o CFF, os medicamentos são o principal agente causador de intoxicação em seres humanos desde 1994. "A automedicação levou para o hospital mais de 60 mil pessoas de 2010 a 2015, de acordo com pesquisa do Ministério de Saúde”. (Guedes, 2017).

E com o surgimento da pandemia causada pelo vírus da COVID-19 este número só tende a aumentar. No ano de 2019 as vendas da hidroxicloroquina, mais que dobraram, passando de 963 mil para 2 milhões de unidades, no caso da ivermectina o aumento foi ainda maior, atingindo 557,26\%, em 2020, apesar de nenhuma agência ou instituição internacional ter recomendado o uso da ivermectina para o tratamento do indivíduo com COVID-19. Mesmo com a variedade de estudos realizados e publicados, a maioria apresenta pequenos tamanhos amostrais, em contextos distintos, com resultados que variam entre si, a 
Organização Mundial de Saúde- OMS (2021) declara que "a evidência atual sobre o uso de ivermectina para tratar pacientes com COVID-19 é inconclusiva.

Durante o período de pandemia, os conselhos de farmácia realizaram varias campanhas para a consientização da população diante os riscos da automedicação. Esta conscientização se tornou mais necessária após a divulgação de dados de um estudo realizado a pedido dos conselhos, a pesquisa realizada pela empresa IQVIA, constatou um aumento significativo nas vendas, nos três primeiros meses de 2020, quando aumentaram os casos da enfermidade, de alguns medicamentos relacionados à COVID-19, comparado ao mesmo período do ano passado. Como apresentado na Tabela 1:

Tabela 1 - Aumeto do consumo de medicamentos entre os anos de 2019 e 2020.

\begin{tabular}{llll}
\hline \multicolumn{1}{c}{ Medicamento/Princípio Ativo } & \multicolumn{1}{c}{ Jan - Mar/2019 } & \multicolumn{1}{c}{ Jan - Mar/2020 } & \% \\
\hline Hidroxicloroquina Sulfato & 231.546 & 388.829 & $67,93 \%$ \\
\hline Ibuprofeno & 15.010 .195 & 14.615 .066 & $-2,63 \%$ \\
\hline Paracetamol & 11.150 .452 & 19.774 .819 & $77,35 \%$ \\
\hline Dipirona Sódica & 30.226 .256 & 46.716 .599 & $54,56 \%$ \\
\hline Cole calciferol (Vitamina D) & 4.440 .289 & 6.019 .038 & $35,56 \%$ \\
\hline Ácido Ascórbico (Vitamina C) & 9.327 .016 & 26.116 .340 & $180,01 \%$ \\
\hline
\end{tabular}

Fonte: Adaptada do Conselho Federal de Farmácia (2020).

Estes dados são reflexo do medo da população em relação ao vírus e também da influência negativa da mídia para um suposto tratamento da COVID-19. O Conselho de Farmácia ressalta que ambos medicamentos oferecem riscos a saúde, mesmo dispensados de prescrição podem causar danos ao indivíduo, especialmente se forem usados sem indicação ou orientação de um profissional. No caso da hidroxocloroquina, medicamento utilizado para o tratamento de doenças como o lúpus eritematoso, seu uso inapropiado pode "causar problemas na visão, convulsões, insônia, diarreias, vômitos, alergias graves, arritmias e até parada cardíaca". Segundo o presidente do CFF, a recomendação e que os farmacêuticos continuem a seguir as orientações da ANVISA (2020):

A nossa recomendação é que os farmacêuticos continuem observando as recomendações da Anvisa e as boas práticas farmacêuticas para realizar as dispensações desses medicamentos, e que orientem os usuários, pois a desinformação é um inimigo tão poderoso quanto o novo coronavírus.

O uso irracional de medicamentos tende a ser maior entre os idosos, pois se trata de um grupo de pessoas com maiores problemas de saúde. Rocha e Miceli (2017), na sua pesquisa, informam que o Brasil é o país que mais consome medicamentos sem prescrição médica em todo o mundo, nesse sentido, destaca- se a população idosa, que por conta da idade tendem a ficar mais doentes. Durante a pandemia, esses dados também são alarmantes, dado que os idosos tendem a ser mais vulneráveis aos sintomas da doença e por conta da falta de informação ou pela influência midiática tendem a buscar medicamentos sem prescrições médicas (Oliveira et al., 2021). Silva e Gonzales. (2021 p. 138) abordam os principais fatores que contribuem para o automedicamento da população idosa:

A automedicação é um fenômeno social e não apenas farmacológico pelo fato de manifestar-se a partir de comportamentos sociais de determinados grupos, como por exemplo a população idosa, estando relacionada a costumes, tradições e crenças populares e, a partir deste contexto, se faz presente na forma em que boa parte da população brasileira consome medicamentos. Por ser uma prática comum, apresentando em determinadas situações aspectos positivos, é propagada também através do reforço social. 
Para Carvalho e Guimarães (2020), todas essas influências midiáticas que contribuem para a automedicação vão de desencontro com a ciência, causando na população um certo "negacionismo científico" que contribui drasticamente com o crescimento de infectados pelo vírus.

A negação da ciência (além da desinformação) talvez seja um dos principais fatores que, no cenário da pandemia, influencie as pessoas a se automedicarem e, assim, correrem riscos com a saúde, uma vez que se cria uma falsa sensação de proteção contra a COVID-19 que, associada à descrença científica, culminam no desrespeito do isolamento social e, consequentemente, no crescimento da curva de infectados (Carvalho \& Guimarães, 2020 p. 3 ).

Diante de uma pandemia, as informações repassadas ou compartilhadas podem causar grandes problemas, principalmente quando se trata de uma notícia falsa. (Falcão \& Souza, 2020) abordam que a fake news não ocorreram apenas no Brazil. Em todo o mundo houve a disseminação de notícias falsas em relação a COVID-19. O que acabou chamando mais atenção no Brasil foi o fato de que muitas das informações falsas a respeito da doença foram disseminadas pelo próprio presidente da república, e por seus apoiadores, o que acabou aumentando ainda mais a gravidade ao fenômeno das fake news no país, visto que o mesmo possui a posição de chefe de Estado e alcança uma maior visibilidade - inclusive na rede nacional de rádio e televisão. Do negacionismo quanto à gravidade da pandemia à divulgação massiva de remédios sem qualquer comprovação científica de eficácia comprovada, como o exemplo do "Kit COVID, o presidente pode ter contribuído de forma secundária para a disseminação da doença no Brasil, já que muitos acreditavam na eficácia do suposto tratamento. Entretanto, vale ressaltar a importância do trabalho jornalístico sério e ético diante desse cenário tão delicado - tanto dos profissionais quanto dos veículos de imprensa -, bem como a atuação das agências de checagem de informações.

Um levantamento importante, realizado pela comunidade de mobilização on-line Avaaz, mostra que pelo menos sete em cada 10 brasileiros acreditaram em pelo menos um conteúdo falso relacionado a pandemia, o plicativo que aparece como maior propagador das fake news é o WhatsApp, 59\% dos intrevistados relatam ter tido acesso a pelo menos uma noticia falsa recibida por meio do aplicativo. "Buscando um meio para combater as fake news sobre saúde, Ministério da Saúde de forma inovadora, disponibilizou um número de WhatsApp para que a população envie mensagens, o canal é um espaço voltado para receber informações virais, que serão apuradas pelas áreas técnicas e respondidas oficialmente se são falsas ou mentirosas." (Ministério da Saúde, 2021).

Para o leitor, é importante verificar a veracidade da notícia antes de tomar qualquer atitude, principalmente quando se envolve saúde como a prática de automedicação (Gomes et al., 2020). Carvalho e Guimarães (2020) abordam a importância da “alfabetização digital” para que todos consigam distinguir as notícias verdadeiras das falsas, entretanto, nos dias atuais a ciência se torna importantíssima para contrapor notícias falsas e desinformações.

Todo esse cenário de negacionismo da ciência e utilização de automedicação durante a pandemia da COVID - 19 indica a necessidade de nos debruçarmos sobre essa questão a fim de estudarmos por meio de uma Pesquisa Bibliográfica os riscos de tais atos em plena pandemia.

\section{Considerações Finais}

Diante disto, a influencia das notícias propagadas pelos meios de comunicação como jornais, Whatsapp e muitas outras redes sociais vem contribuindo de forma negativa para a automedicação durante a pandemia do novo coronavírus, fazendo com que a população use de forma indiscriminada medicamentos que não possuem nenhuma evidência científica para tratamento da COVID-19. Vale ressaltar que nem tudo que está disponível como informação veiculada nos diversos tipos de meios de comunicação corresponde a um fato, o cenário de pandemia favoreceu e muito a disseminação de informações errôneas. 
O farmacêutico tem um papel fundamental para a orientação sobre os medicamentos prezando pela saúde e bem estar dos pacientes visto que automedicação pode influenciar de forma negativa a saúde do indivíduo. Diante do cenário de pandemia cabe ao profissional atuar com o intuito de triar muitas informações falsas sobre tratamentos, o mesmo pode atuar com palestras educativas, conscientizando à população da importância de sempre buscar uma orientação de um profissional da saúde. Através de campanhas educativas, realizadas e enfatizadas rotineiramente para a sociedade a fim de minimizar a prática da automedicação.

Considera-se, portanto, importante que futuramente pesquisadores realizem estudos relacionados aos impactos trazidos para saúde dos indivíduos devido o aumento da automedicação no período da pandemia, relatando assim as consequências que este hábito trouxe e também observar se continua tendo um aumento ou a população diminuiu esta prática. Estudos podem trazer também novas informações sobre a propagação das fakes news e meios adotados para que a população saiba diferenciar notícias verdadeiras das notícias falsas.

\section{Referências}

Amaral, O., Veiga, N., Nelas, P., Coutinho, E., \& Chaves, C. (2019). Automedicação na comunidade: um problema de saúde pública. International Journal of Developmental and Educational Psychology. Revista INFAD de Psicología., 3(1), 423-432.

Brasil. (2021). Comunicação do CFF; Busca de fórmulas milagrosas contra a COVID-19 continua impulsionando vendas de medicamentos.

Brasil. (2021). Comunicação do CFF; Levantamento mostra como o medo da COVID-19 impactou venda de medicamentos, 2020.

Bispo, E. J. (2020). EEP: Escola de Educação Permanente; Automedicação e os riscos à saúde em tempos de COVID-19. Website da EEP - Escola de Educação Permanente do Hospital das Clínicas da FMUSP. https://eephcfmusp.org.br/portal/online/automedicacao-riscos-saude-COVID19/.

Carvalho, W., \& Guimarães, Á. S. (2020). Desinformação, Negacionismo e Automedicação: a relação da população com as drogas “milagrosas” em meio à pandemia da COVID-19. InterAmerican Journal of Medicine and Health, 3.

Da Silva, E. L Jr., \& Gonzalez, L. F. C. Automedicação e Efeitos Psicológicos em Idosos Durante o Isolamento Social. Automedicação e Efeitos Psicológicos em Idosos Durante o Isolamento Social, 1-388.

De Fátima Souza, A., Pinheiro, A. C., Porto, J. M., Costa, J. S. C., \& Dias, R. C. N. (2021). COVID-19: Automedicação de indivíduos psicologicamente afetados. Brazilian Journal of Development, 7(1), 2718-2731.

De Oliveira Rocha, A. P. C., Miceli, B. C. (2017).. A influência da mídia no consumo de medicamentos por idosos em uma drogaria na cidade de ParaopebaMg. Revista Brasileira de Ciências da Vida. 5(5).

Dos Santos, J. R. M., Monteiro, L., de Sousa, S. G., \& de Araújo, B. G. (2021). Os riscos da automedicação por hidroxicloroquina frente a Pandemia de COVID19 The risks of hydroxychlorochine self-medication in front of the COVID-19 Pandemic. Brazilian Journal of Health Review, $4(3), 11185-11204$.

Falcão, P., \& Souza, A. B. D. (2021). Pandemia de desinformação: as fake news no contexto da COVID-19 no Brasil.

Ferreira, I. S., \& de Carvalho, C. J. S. (2021). A influência da propaganda de medicamentos na prática da automedicação: um problema de saúde pública. Brazilian Journal of Development, 7(5), 47642-47652.

Freitas, A. R. R., Napimoga, M., \& Donalisio, M. R. (2020). Análise da gravidade da pandemia de COVID-19. Epidemiologia e serviços de saúde, 29.

Gomes $^{1}$, A. H. D., Rocha ${ }^{1}$, A. K. A., de Sousa Viana1, T., \& Bachur, T. P. R. (2020). Riscos da automedicação na pandemia por COVID-19: O dilema entre informações midiáticas e científicas. copyright $@$ editora amplla editor chefe: leonardo pereira tavares, 40.

Guedes, Aline. (2017). A automedicação pode ter graves consequências. Agência Senado.

Imoto, A. M., Gottems, L. B. D., Castelo Branco, H. P., Santana, L. A., Monteiro, O. L. R., Fernandes, S. E. S., \& Amorim, F. F. (2020). Cloroquina e Hidroxicloroquina no tratamento da COVID-19: Sumário de Evidências. Comunicação em Ciências da Saúde.

Melo, J. R. R., Duarte, E. C., Moraes, M. V. D., Fleck, K., \& Arrais, P. S. D. (2021). Automedicação e uso indiscriminado de medicamentos durante a pandemia da COVID-19. Cadernos de Saúde Pública, 37, e00053221.

Miguel, L. C. B., \& Carvalho, C. J. S. (2021). O impacto das fake news e a sua influência na automedicação na COVID $19,5,145$.

Ministério de Saúde. (2020). Agência Nacional de Vigilância Sanitária. Resolução $\rightarrow$ RDC no 351, DE 20 DE MARÇO DE 2020. Brasília.

Ministério de Saúde. (2021). 1 ano Saúde sem fakenews. 
Research, Society and Development, v. 10, n. 13, e53101321015, 2021

(CC BY 4.0) | ISSN 2525-3409 | DOI: http://dx.doi.org/10.33448/rsd-v10i13.21015

Naves, J. D. O. S., Castro, L. L. C. D., Carvalho, C. M. S. D., \& Merchán-Hamann, E. (2010). Automedicação: uma abordagem qualitativa de suas motivações. Ciência \& Saúde Coletiva, 15, 1751-1762.

Oliveira, J. V. L., da Costa, F. B., do Nascimento Porfirio, V., da Silva, M. M. M., da Silva, N. C., de França, A. M. M., \& da Silva Filho, L. S. (2021). A automedicação no período de pandemia de COVID-19: Revisão integrativa. Research, Society and Development, 10(3), e58610313762-e58610313762.

Pizzani, L., da Silva, R. C., Bello, S. F., \& Hayashi, M. C. P. I. (2012). A arte da pesquisa bibliográfica na busca do conhecimento. RDBCI: Revista Digital de Biblioteconomia e Ciência da Informação, 10(2), 53-66.

Rubert, C., \& Deuschle, R. A. N. (2020). Assistência farmacêutica durante a pandemia da COVID-19: revisão da literatura. Revista Interdisciplinar De Ensino, Pesquisa E Extensão, 8(1), 255-268.

Trotta, Raphael. (2020, setembro, 18). Os riscos das notícias falsas sobre saúde: Medicina S/A, https://medicinasa.com.br/tag/raphael-trotta/

Wiese, L., Ostrovski, E. G., Keil, E. S., Keunecke, F. R., Barboza, J., \& Danski, V. R. R. (2020). Projeto de Extensão riscos da automedicação: relato de experiências em educação em saúde. Extensão Tecnológica: Revista De Extensão Do Instituto Federal Catarinense, 7(13), 64-88. 